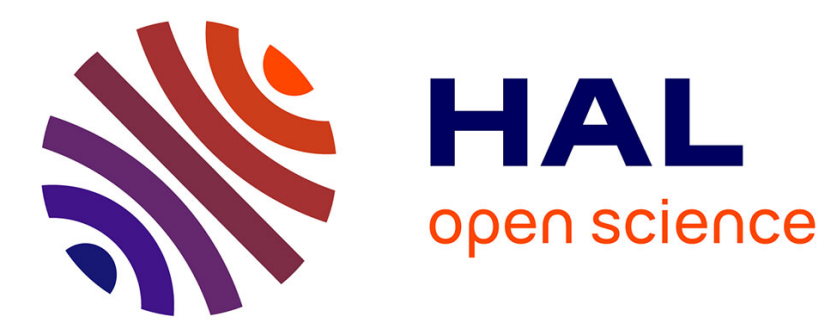

\title{
Transient frictionless contact of a rough rigid surface on a viscoelastic half-space
}

Romain Bugnicourt, Philippe Sainsot, Nicolas Lesaffre, Antonius A. Lubrecht

\section{To cite this version:}

Romain Bugnicourt, Philippe Sainsot, Nicolas Lesaffre, Antonius A. Lubrecht. Transient frictionless contact of a rough rigid surface on a viscoelastic half-space. Tribology International, 2017, 10.1016/j.triboint.2017.01.032 . hal-01446969

\section{HAL Id: hal-01446969 \\ https://hal.science/hal-01446969}

Submitted on 31 Jan 2017

HAL is a multi-disciplinary open access archive for the deposit and dissemination of scientific research documents, whether they are published or not. The documents may come from teaching and research institutions in France or abroad, or from public or private research centers.
L'archive ouverte pluridisciplinaire HAL, est destinée au dépôt et à la diffusion de documents scientifiques de niveau recherche, publiés ou non, émanant des établissements d'enseignement et de recherche français ou étrangers, des laboratoires publics ou privés.

\section{(1) (1) $\$$}

Distributed under a Creative Commons Attribution - NonCommercial - NoDerivatives 44.0 


\title{
Transient frictionless contact of a rough rigid surface on a viscoelastic half-space.
}

\author{
R. Bugnicourt ${ }^{\mathrm{a}, \mathrm{b}, *}$, P. Sainsot ${ }^{\mathrm{a}}$, N. Lesaffre ${ }^{\mathrm{b}}$, A.A. Lubrecht $^{\mathrm{a}}$ \\ ${ }^{a}$ Univ Lyon, INSA-Lyon, CNRS UMR5259, LaMCoS, F-69621, France \\ ${ }^{b}$ Manufacture Française de Pneumatiques Michelin, F-63040 Cedex 9, France
}

\begin{abstract}
The contact problem of a rough, rigid surface with an elastic or viscoelastic incompressible semi-infinite body is studied in this paper. The problem is solved using a Boundary Element Method coupled with a conjugate gradient method. Viscoelasticity is taken into account with a 'State Variable' approach, making it possible to tackle the transient problem efficiently. The results are compared against Persson's theory of transient viscoelastic contact, showing good agreement in terms of contact area ratio and friction coefficient.
\end{abstract}

Keywords: Sliding contact; Boundary element method; Roughness

\section{Introduction}

Predicting the contact surface and friction of rubber materials on a road is of critical interest for the tire industry. This particular contact problem is influenced by the viscoelasticity of rubber and by the multi-scale surface roughness of the road.

There are two main analytical approaches for the contact problem. The first one is a multiasperity approach, initiated by the pioneering work of Greenwood and Williamson [I]. For a Hertzian contact between a sphere and an elastic half-space analytical solutions exist for both a normal and a tangential (friction) force [2]. The idea of a multi-asperity approach is to generalize these relations by considering an infinite distribution of spherical indentors of different height and different radii. The radius and height distribution are derived from the summit height distribution and the summit curvature distribution of the rough surface under consideration. The approach can be extended to viscoelastic frictional contacts as in [3]. However multi-asperity approaches suffer from a major weakness: they do not account for the influence of the indentors on each other, so they are only valid at small normal loads

The second approach is the one initiated in [4]. Normal displacements of the half space are supposed to follow the same Power Spectral Density as the rough surface. From this hypothesis the normal contact problem is solved and the friction is deduced in the case of a viscoelastic half

\footnotetext{
${ }^{*}$ Corresponding author, email address: romain.bugnicourt@insa-lyon.fr

${ }^{0}$ (C)2017. This manuscript version is made available under the CC-BY-NC-ND 4.0 license http:// creativecommons.org/licenses/by-nc-nd/4.0/

It has been accepted for publication in Tribology International. The published version is available at the following DOI: $10.1016 /$ j.triboint.2017.01.032
} 
space sliding at constant speed. This model is analytically exact for full contact but is less precise as the contact ratio decreases. This defect was modified in [5] to improve results for low contact ratios and has been widely tested by numerical simulations for both elastic [6] and viscoelastic contact [ [ $]$ ]. Shortly after his 2001 article, Persson adapted the model in [8] to handle transient sliding. Transient sliding is of interest in tire modeling as the rubber resides in the contact zone for only milliseconds and slides only millimeters. Under such conditions steady state modeling is not accurate. It should be highlighted that in Persson-like approaches the friction is always deduced from the viscoelastic losses in the half space and that no contact friction is taken into account.

A review and a comparison of the two approaches is available in [9].

To overcome the limitations of analytical solutions, numerical simulations are necessary. Numerical simulations are limited by the fact that a very fine mesh is necessary to handle the different length scales of the surface roughness. A 3-dimensional mesh would be excessively big. Using the assumption that the rubber block is very large compared to the size of the simulation and undergoing small strains, simplifies the problem. Using Green's functions, the surface displacements are directly related to the surface stresses, so only the surface needs to be meshed.

Analyzing 2 or 3 length scales of surface roughness still requires more than a million degrees of freedom and consequently long computation times. Typical roads have a surface roughness over more than 6 length scales, which means the simulations should be very fast to take into account as many length scales as possible. Using a multi-level multi-summation technique Brandt and Lubrecht [IIV] obtain a reduction of the computational cost from $O\left(N^{2}\right)$ to $O(N \log (N)), N$ being the number of degrees of freedom (DOF). A number of other techniques have since been developed. Using Fast Fourier Transforms (FFT) as in [II] allows the same reduction provided the mesh is regular and periodic. A slightly slower extension to non-periodic problems is found in [[2]. A comparison between multi-level and FFT methods is given in [13]. Another approach is to use a mesh smart enough to reduce the number of DOF without impacting precision. This is achieved by refining the mesh at the edges of contact clusters only and keeping a relatively coarse mesh in the inner part. This 'Active Sets' method are developed in [14][15]. Molecular Dynamics solvers were also developed: GFMD [16] [17] and RMD [7].

This paper uses Fast Fourier Transforms with a conjugate gradient iteration scheme.

\section{Numerical model}

Firstly, the contact between an elastic half space and a rigid body without friction is considered. The relation between surface pressure $P$ and surface normal displacement $U_{z}$ was first found by Boussinesq [18] and is given by:

$$
U_{z}(x, y)=\frac{1-\nu}{2 \pi G} \iint_{\Omega} \frac{P(\xi, \eta)}{\sqrt{(x-\xi)^{2}+(y-\eta)^{2}}} d \xi d \eta
$$

$G$ is the shear modulus, $\nu$ the Poisson ratio, $x, y, \xi$ and $\eta$ are spatial variables.

Using the Boussinesq equations Love [I.,] found the normal displacements for a uniformly distributed pressure over a polygonal region. Discretizing the surface into a uniform mesh with square cells and using these results leads to Equation [2.

$$
U_{z}(i, j)=\frac{1}{G} \sum_{i^{\prime}, j^{\prime}} A_{z z}\left(i-i^{\prime}, j-j^{\prime}\right) P\left(i^{\prime}, j^{\prime}\right)
$$


$A_{z z}$ is the Influence Coefficient matrix, which is detailed in [20] and depends on the mesh size. Equation $\nabla$ is a convolution and therefore can be computed very efficiently in Fourier space. Here the problem is considered to be periodic, as periodic random rough surfaces will be used. Using FFT and non-periodic surfaces is also possible with the appropriate zero-padding [12].

Solving the contact problem means finding the pressure in the contact zone satisfying:

$$
\begin{aligned}
P & \geq 0 \\
U_{z}-H & \geq 0 \\
U_{z} & =H \text { in the contact zone } \\
P \quad & =0 \text { outside the contact zone }
\end{aligned}
$$

$U_{z}$ is the normal displacement calculated from the pressure (Equation $\left.\nabla\right)$ and $H$ is the rigid substrate height map.

An efficient and easy to implement way to find $P$ is to use an iterative procedure, such as the conjugate gradient method [21], [14], [15]. For a contact problem the procedure is slightly different from a classical conjugate gradient as the contact surface is not constant. One solution is to solve for a fixed contact surface and change the contact surface by removing the points with negative pressures and including the points where the two surfaces interpenetrate. Then solve again and continue the loop while the contact surface is not constant. This procedure guarantees the existence and uniqueness of the solution and convergence [20]. It is rather slow though, as the Conjugate Gradient has to be executed several times. The usual solution to avoid this is to update the contact surface within the Conjugate Gradient algorithm, between each iteration. This is the solution chosen here.

\subsection{Viscoelasticity}

Discretizing time into small time steps makes it possible to model the transient response of a viscoelastic half space.

The Zener, or Standard Linear Solid viscoelastic model is used. It can be represented as a spring and a dashpot connected in parallel (elastic shear modulus $G_{1}$, viscosity $\eta_{1}$ ), connected in series to another spring (modulus $G_{0}$ ) - see Figure Ш.

Rubber is considered as an incompressible material. An incompressible viscoelastic material

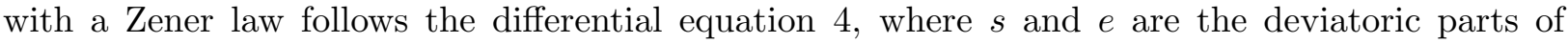
the stress and strain tensors, the dot denotes a time derivative. This differential formulation for viscoelasticity is strictly equivalent to the integral formulation as mentioned in [ $[22]$.

$$
s\left(1+\frac{G_{1}}{G_{0}}\right)+\frac{\eta_{1}}{G_{0}} \dot{s}=2 G_{1} e+2 \eta_{1} \dot{e}
$$

Now let us consider a contact problem with a normal pressure imposed on an incompressible viscoelastic half space. This kind of problem can be treated using functional equations [22]. In the Laplace domain, the problem is equivalent to an elastic contact problem, which means it is possible to use analytical elastic solutions, in particular the Boussinesq potential. Going back to the real domain, the viscoelastic Boussinesq equation is given by Equation 5 . 


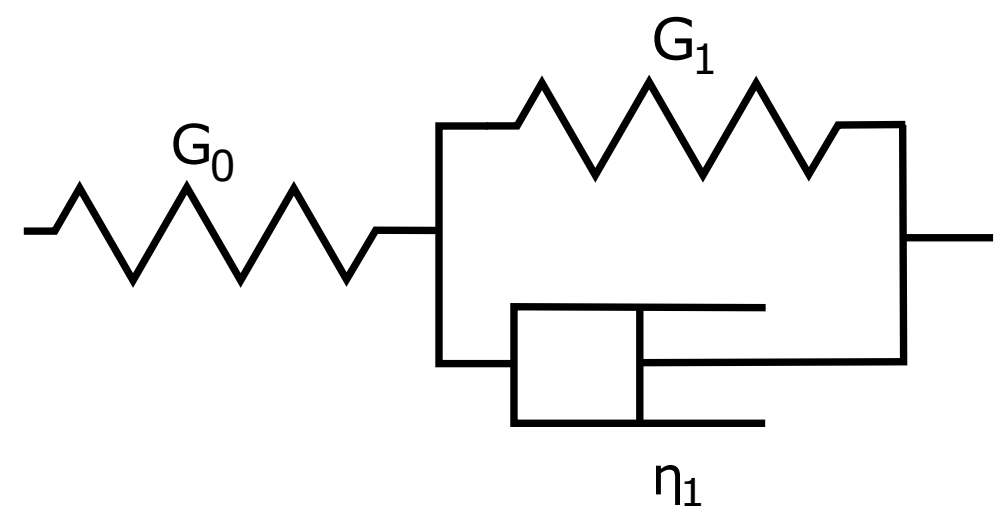

Figure 1: Representation of the Standard Linear Solid. $G_{0}$ and $G_{1}$ are the spring stiffness, $\eta_{1}$ the dashpot damping coefficient.

$$
\begin{gathered}
\iint_{\Omega} \frac{1}{4 \pi \rho}\left(P(\xi, \eta)\left(1+\frac{G_{1}}{G_{0}}\right)+\frac{\eta_{1}}{G_{0}} \dot{P}(\xi, \eta)\right) d \xi d \eta=G_{1} U_{z}(x, y)+\eta_{1} \dot{U}_{z}(x, y) \\
\text { with } \rho=\sqrt{(x-\xi)^{2}+(y-\eta)^{2}}
\end{gathered}
$$

For an implementation in BEM, Equation 1 is discretized in space and leads to Equation [6, where $i, i^{\prime}, j$ and $j^{\prime}$ denote the row and column of the considered mesh cell.

$$
\begin{gathered}
\sum_{i^{\prime}, j^{\prime}} A_{z z}\left(i-i^{\prime}, j-j^{\prime}\right) P\left(i^{\prime}, j^{\prime}\right)\left(1+\frac{G_{1}}{G_{0}}\right)+\frac{\eta_{1}}{G_{0}} \sum_{i^{\prime}, j^{\prime}} A_{z z}\left(i-i^{\prime}, j-j^{\prime}\right) \dot{P}\left(i^{\prime}, j^{\prime}\right) \\
=G_{1} U(i, j)+\eta_{1} \dot{U}(i, j)
\end{gathered}
$$

Discretizing time $t$ in the previous equation and removing the sum notations leads to:

$$
A_{z z} P_{t+\delta t}\left(1+\frac{G_{1}}{G_{0}}\right)+\frac{\eta_{1}}{G_{0}} A_{z z} \frac{\Delta P}{\Delta t}=G_{1} U_{t+\delta t}+\eta_{1} \frac{\Delta U}{\Delta t}
$$

$\Delta P$ and $\Delta U$ are the variation of pressure and displacement between time step $t$ and time step $t+\Delta t$.

A generalized Zener model is a combination of different Zener models connected in parallel, plus a branch with just one spring $G_{\infty}$. It is necessary to use it for rubber in order to model its behavior over a large range of frequencies. The displacement in each branch is the same and the pressures in each branch add up. Each branch $k$ follows Equation $\mathbf{\square}$. It leads to Equation $\mathbf{8}$.

$$
\begin{gathered}
U_{t+\Delta t}\left[G_{\infty}+\sum_{k} \frac{G_{1}^{k}+\frac{\eta_{1}^{k}}{\Delta t}}{1+\frac{G_{1}^{k}}{G_{0}^{k}}+\frac{\eta_{1}^{k}}{G_{0}^{k} \Delta t}}\right]=A_{z z} \sum_{k} P_{t+\Delta t}^{k}+U_{t} \sum_{k} \frac{\frac{\eta_{1}^{k}}{\Delta t}}{1+\frac{G_{1}^{k}}{G_{0}^{k}}+\frac{\eta_{1}^{k}}{G_{0}^{k} \Delta t}} \\
-\sum_{k} A_{z z} P_{t}^{k} \frac{\frac{\eta_{1}^{k}}{G_{0}^{k} \Delta t}}{1+\frac{G_{1}^{k}}{G_{0}^{k}}+\frac{\eta_{1}^{k}}{G_{0}^{k} \Delta t}}
\end{gathered}
$$


It is then possible to use the 'elastic' Conjugate Gradient contact solver using $P^{\prime}, U_{z}^{\prime}$ and $H^{\prime}$

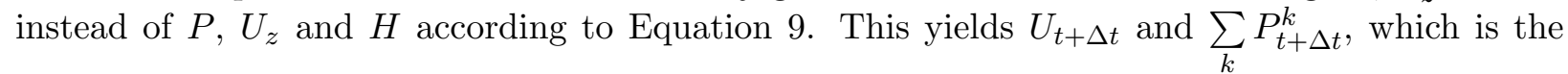
total pressure acting on the surface.

$$
\begin{aligned}
P^{\prime}= & \sum_{k} P_{t+\Delta t}^{k} \\
U_{z}^{\prime}= & A_{z z} P^{\prime} \\
H^{\prime}= & H\left[G_{\infty}+\sum_{k} \frac{G_{1}^{k}+\frac{\eta_{1}^{k}}{\Delta t}}{1+\frac{G_{1}^{k}}{G_{0}^{k}}+\frac{\eta_{1}^{k}}{G_{0}^{k} \Delta t}}\right]-U_{t} \sum_{k} \frac{\frac{\eta_{1}^{k}}{\Delta t}}{1+\frac{G_{1}^{k}}{G_{0}^{k}}+\frac{\eta_{1}^{k}}{G_{0}^{k} \Delta t}} \\
& +\sum_{k} A_{z z} P_{t}^{k} \frac{\frac{\eta_{1}^{k}}{G_{0}^{k} \Delta t}}{1+\frac{G_{1}^{k}}{G_{0}^{k}}+\frac{\eta_{1}^{k}}{G_{0}^{k} \Delta t}}
\end{aligned}
$$

It should be highlighted that it is necessary to compute and to store the state variables $A_{z z} P_{t}^{k}$ for each branch of the generalized Zener model using Equation $\mathbf{7}$. This makes the viscoelastic solver more memory intensive than the elastic one.

Ideally, the time steps should be small compared to the smallest characteristic time $\tau^{k}=$ $\frac{\eta_{1}^{k}}{G_{0}^{k}+G_{1}^{k}}$ of the generalized Zener model.

The integral formulation of viscoelasticity as introduced in [2:3] and used for contact simulations in [24], [25], [26] and [27] requires to store the pressure map of all the previous time steps . The differential formulation used in the current model (and also in [28]) only requires one state variable map at the previous time step and one state variable map per branch of the generalized Zener model to be stored. This latter approach is called a 'State variable approach' in [2.9].

During sliding, the rigid substrate, which defines the normal displacement input, is translated by one cell in the sliding direction at each step. The duration of the time step is deduced from the velocity and cell length. Translation by more than one cell reduces precision: one might as well use a coarser mesh to speed up the computation. Translation by less than one cell is possible but a careful interpolation of the rigid substrate is required, leading to noticeably longer computing times. Indeed the rigid substrate is defined only on a discrete number of points, so when it is translated by less than one cell length the position of the intermediate points needs to be computed. It might be necessary in some cases to avoid too long time steps at low speeds.

\subsection{Hertzian contact}

In order to validate our model, a comparison is made against results from [24] and [15], who both investigated the contact of a rigid sphere with a viscoelastic half space. Carbone et al. [15] focused on the steady-state response while Koumi et al. [24] modeled the transient phase. The viscoelastic material characteristics are $G_{0}=2.75 \mathrm{MPa}, G_{0} / G_{\infty}=10$ and $\tau_{0}=0.01$ where $G_{\infty}=1 /\left(1 / G_{1}+1 / G_{0}\right)$ is the long term shear modulus and $\tau_{0}=\eta_{1} / G_{1}$ is the characteristic time of the creep compliance function. The radius of the sphere is $10 \mathrm{~mm}$. The speed and pressure are 


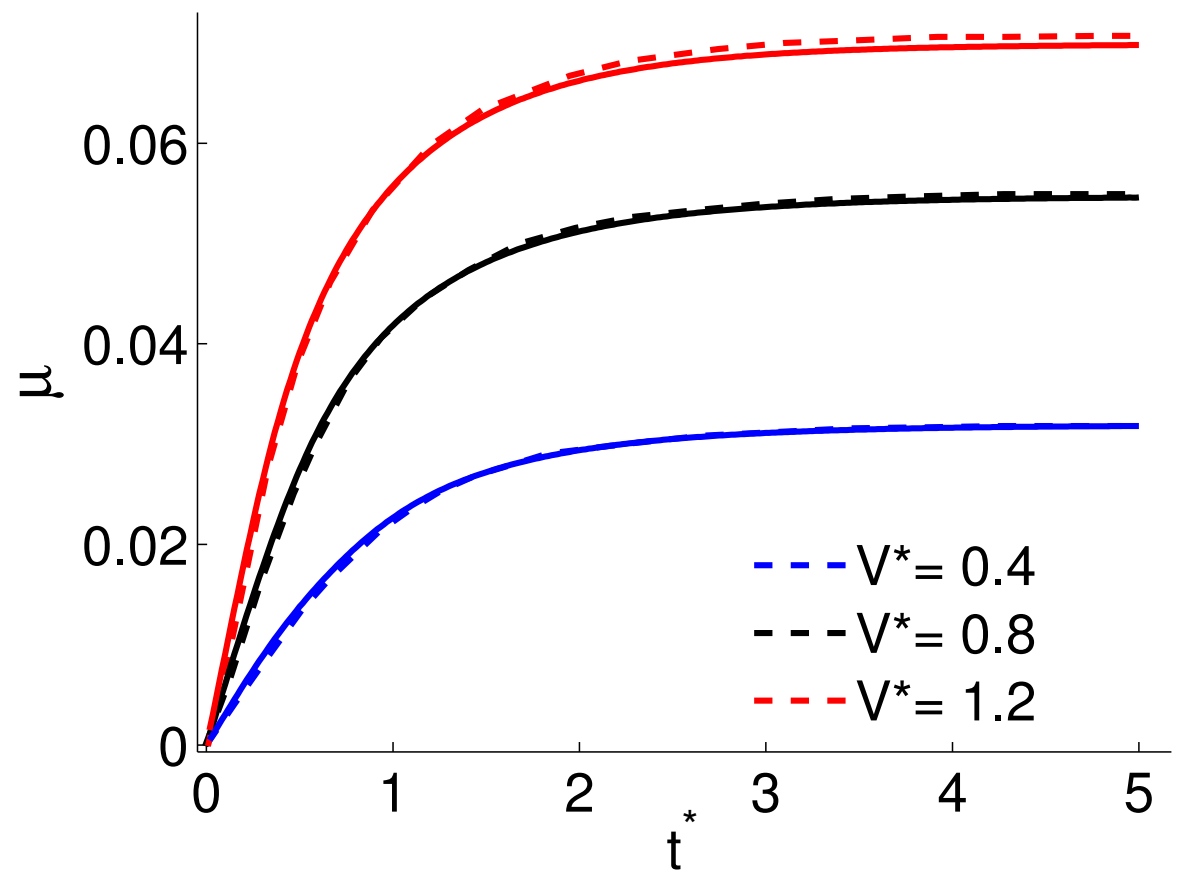

Figure 2: Apparent coefficient of friction for different velocities. Solid lines are for the current model, dashed lines are from [24]

normalized using the Hertzian contact parameters with the instantaneous elastic shear modulus $G_{0}=2.75 \mathrm{MPa}$. The contact radius is $a^{*}$ and the mean pressure in the contact area $p_{0}$. The normalized time and speed are $t^{*}=t / \tau_{0}$ and $V^{*}=V \tau_{0} / a^{*}$.

The values of $G_{0}$ and $G_{\infty}$ are not the same as in [24] as they use a Poisson ratio of $\nu=0.3$ while the current model is for incompressible solids $\nu=0.5$. The ratio $(1-\nu) / G$, which is the only material parameter appearing in Boussinesq's equation, is kept constant so that the results are comparable.

The mesh used is a non-periodic (zero-padded) $1024 \times 1024$ regular mesh and the domain length is $L=10 \mathrm{~mm} \approx 21 a^{*}$ for figures $\square$ and $\mathbf{3}$. The evolution of friction with velocity presented in Figure 四 is calculated with a $512 \times 512$ mesh with $L=12 \mathrm{~mm}$.

The apparent friction coefficient $\mu$ is the absolute value of the tangential force divided by the normal force. The tangential force $F_{t}$ is computed from Equation سل where $S_{c}$ is the contact surface and $H$ the height map of the rigid substrate - in the present case the sphere.

$$
F_{t}=\int_{S_{c}} P \frac{\partial H}{\partial x} d S
$$

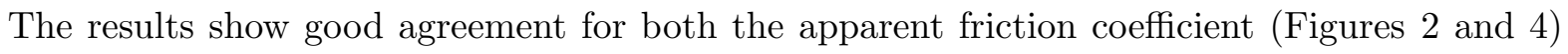
and the pressure distribution (Figure B). 

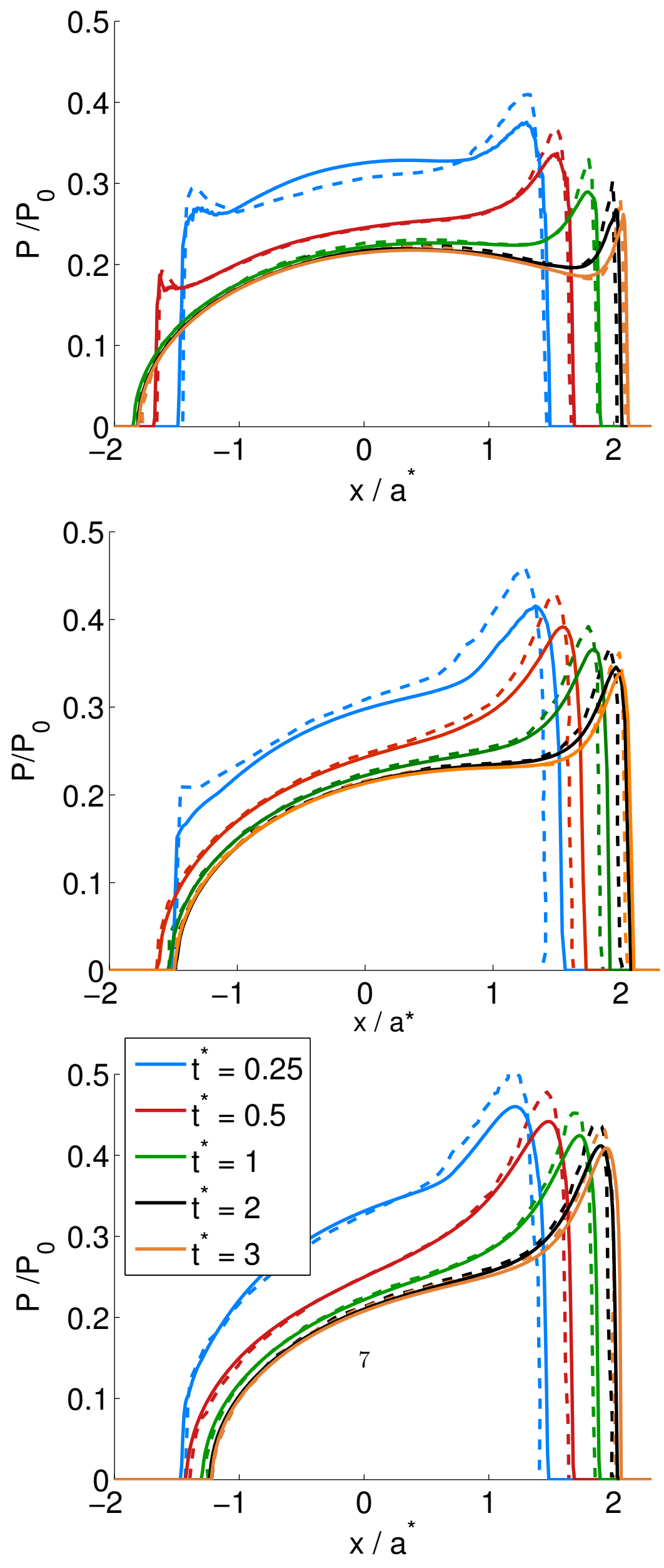


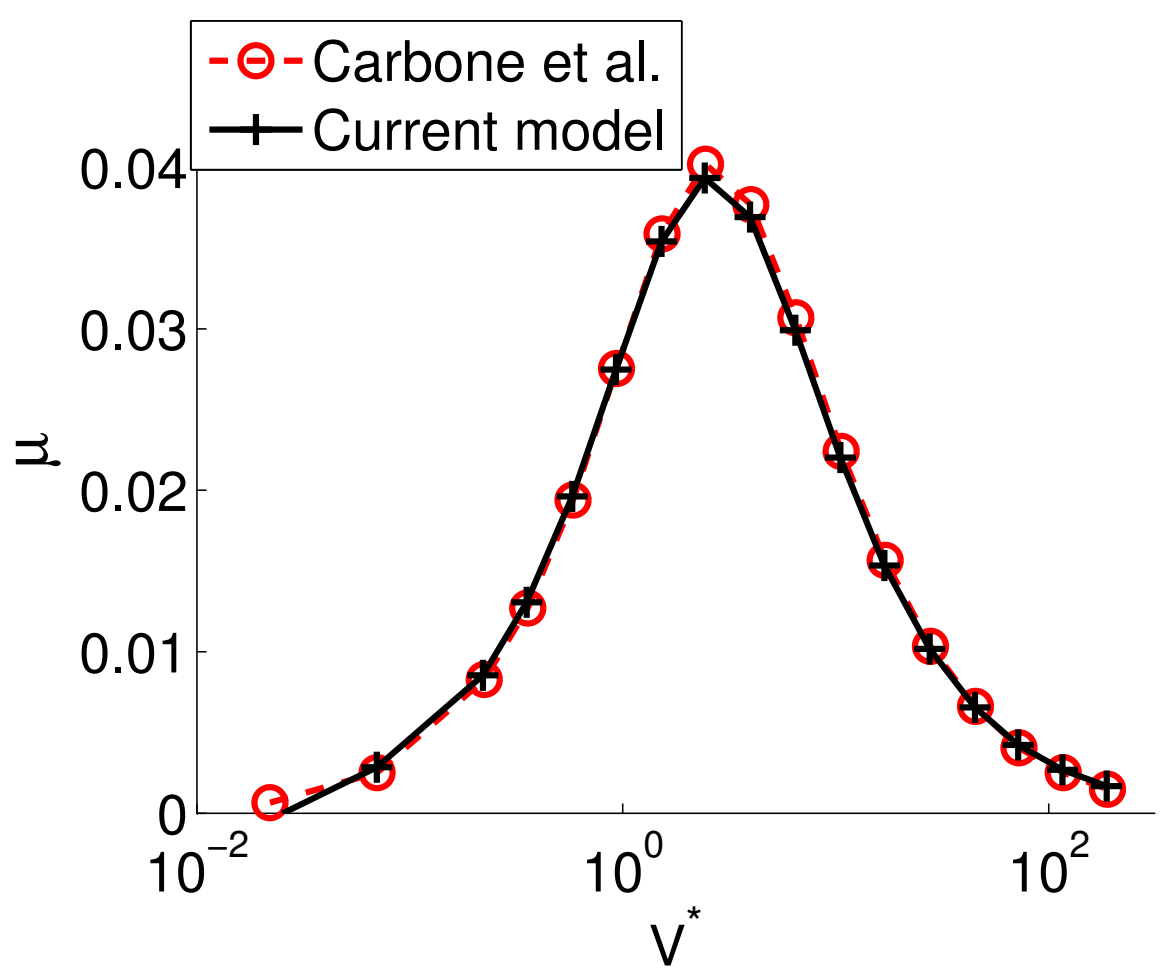

Figure 4: Variation of the apparent friction coefficient with velocity compared to [I.5]

\section{Sliding on a rough surface}

A crucial characteristic of rough surfaces is their Power Spectral Density $\Phi$, which can be defined from the continuous Fourier Transform $\widehat{H}$ of a surface $H$ by:

$$
\Phi\left(k_{x}, k_{y}\right)=\frac{\left|\widehat{H}\left(k_{x}, k_{y}\right)\right|^{2}}{A}
$$

where $A$ is the total surface area and $k_{x}, k_{y}$ are wave vectors. For a discrete surface of $M \times N$ points:

$$
\Phi\left(k_{x}, k_{y}\right)=\frac{A}{(M N)^{2}}\left|\operatorname{FFT}(H)\left(k_{x}, k_{y}\right)\right|^{2}
$$

FFT denotes the discrete Fast Fourier Transform.

Road surfaces can be considered as isotropic random self-affine surfaces, with the following Power Spectral Density. [30]:

$$
\Phi(|\mathbf{k}|)= \begin{cases}C & k_{l}<|\mathbf{k}|<k_{r} \\ C\left(\frac{|\mathbf{k}|}{k_{r}}\right)^{-2(1+H)} & k_{r}<|\mathbf{k}|<k_{s} \\ 0 & \text { otherwise }\end{cases}
$$

Generating random surfaces from a given PSD can be done in several ways, see for example [II] or [3I]. Both these methods are equivalent, though the latter is more general and can also be used to generate non-isotropic surfaces. 


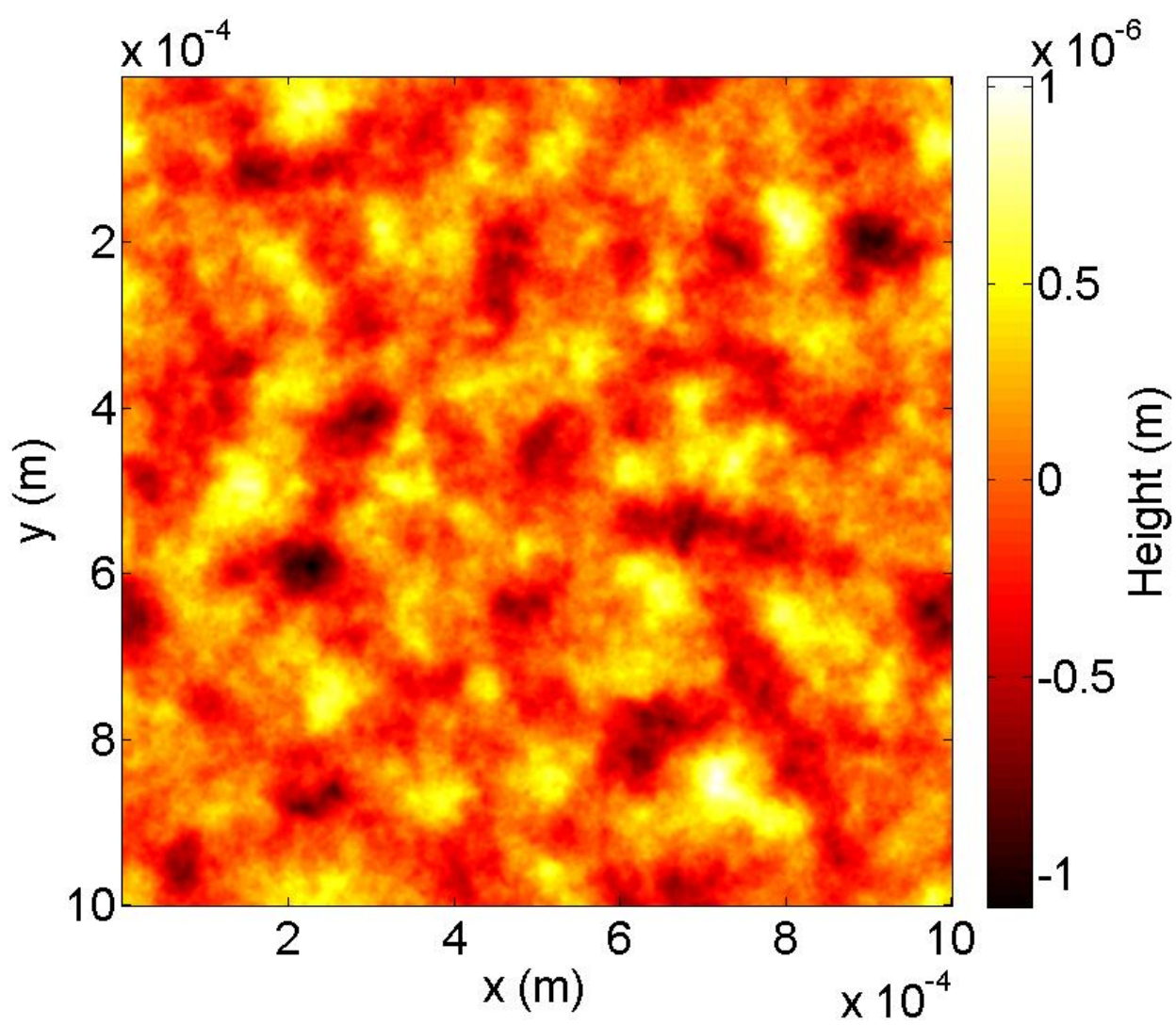

Figure 5: Representation of the rough surface used in the simulations

A particular attention is required concerning the wavenumber cut-offs $k_{l}$ and $k_{s}$. Yastrebov et al. [30] highlighted that the largest wavelength must be small compared to the size of the simulation $\left(k_{l}>2 \pi / L\right)$ in order to achieve meaningful results. Otherwise, the surfaces are non Gaussian, and sometimes non isotropic. The smallest wavelength should also be larger than the size of a cell to achieve a reasonable precision: $k_{s}<2 \pi N / L$.

\subsection{Comparison with Persson's analytical model}

An implementation of Persson's nonstationary model [ $[8]$ is made, modified to include the correction factor introduced in [5] which gives good results at low contact area ratios [6]. This correction factor aims at reducing the predicted friction in the case of partial contact. It depends on a parameter $\gamma \in[0,1]$. When $\gamma=1$ the correction factor has no influence. Friction decreases as $\gamma$ decreases. In the following section, the parameter $\gamma=0.45-$ the same value as in [6].

The examples given in [8] use the following sliding history: rubber is sliding at constant velocity $V_{0}$. At $t=0$ the velocity is changed to $V_{1}$. When $V_{1}>V_{0}$ the contact area decreases, a maximum of the friction coefficient curve $\mu_{\max }$ can occur before the steady state is reached. The steady state friction coefficient is named $\mu_{d y n}$.

Simulations are run comparing the current model to Persson's. The simulation are run on a $L=1 \mathrm{~mm}$ large square with a $N \times N=2048 \times 2048$ mesh. The random surface $H$ has the following properties: $k_{l}=k_{r}=2 \pi * 4 / L, k_{s}=2 \pi * 128 / L, \sqrt{\left\langle(\nabla H)^{2}\right\rangle}=0.02$ and a Hurst exponent 


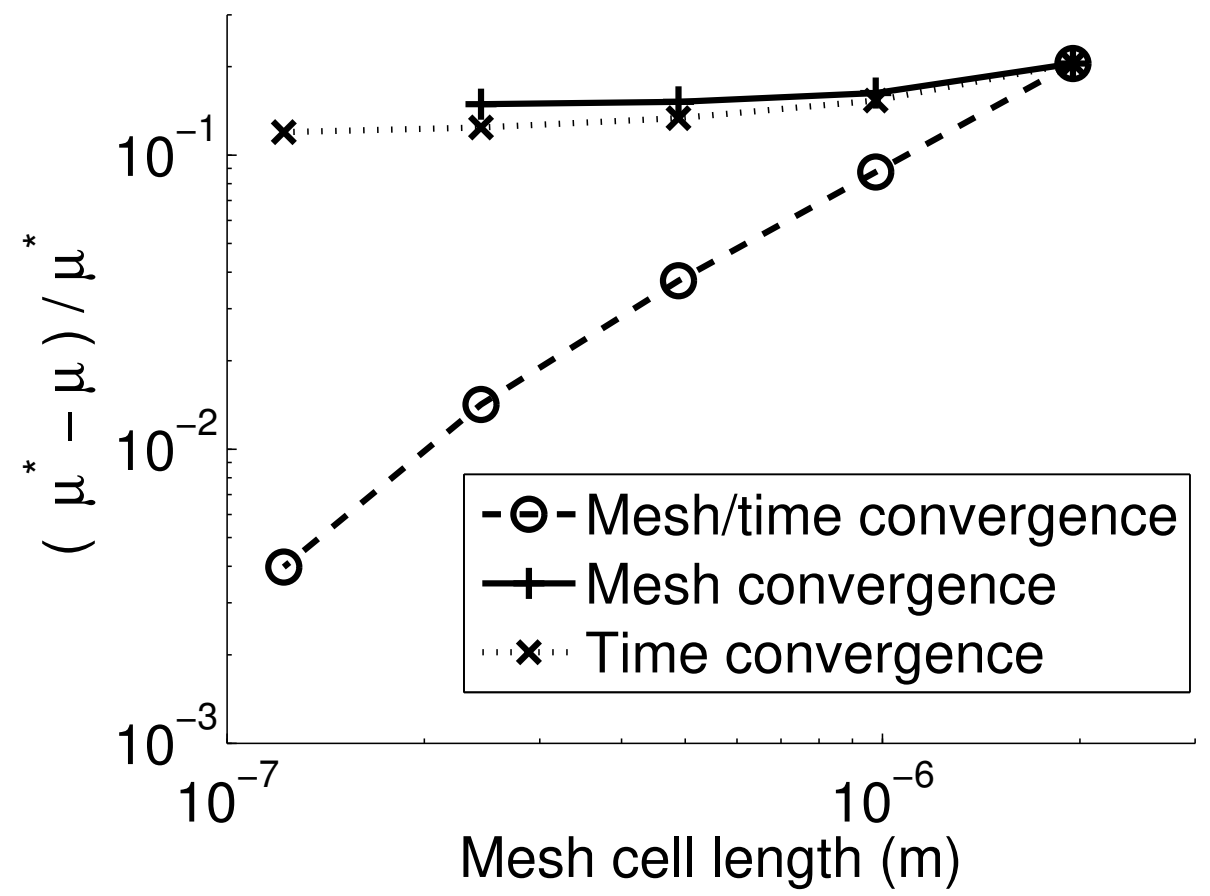

Figure 6: Convergence study for mesh/time refinement, mesh refinement and time refinement. The $\mathrm{x}$ scale for the time convergence curve is the mesh cell length $l$ equivalent to one time step $\Delta t: l=\Delta t / V$

$H=0.9$, which yields $C=1.26 \cdot 10^{-21} \mathrm{~m}^{4}$. The random surface is represented in Figure 5 . The normal pressure is $P_{0}=0.1 \mathrm{MPa}$. In the following section, the initial velocity is $V_{0}=0.01 \mathrm{~m} / \mathrm{s}$ and $V_{1}$ is between 0.1 and $100 \mathrm{~m} / \mathrm{s}$. The material chosen is a typical tire material, modeled using a generalized Zener model. Its characteristics are shown in Figure $\square$.

A convergence study is performed for the lowest speed studied in this paragraph $V=0.1 \mathrm{~m} / \mathrm{s}$, for which the time steps are the longest. Firstly the mesh cell length and time steps are refined accordingly, so the imposed displacement is of one cell length per time step. The precision of the results in terms of $\mu_{\max }$ is $\approx 4 \%$ for the $2048 \times 2048$ mesh compared to the $8192 \times 8192$ mesh. The error is proportional to the mesh cell length to a power of 1.4. In Figure 6 the reference value $\mu^{*}$ is calculated using Equation 때, where $l_{\max }$ and $l_{\min }$ are the maximum and minimum mesh cell length.

$$
\mu^{*}=\frac{\mu_{\max }\left(l_{\max }\right)-\left(l_{\max } / l_{\min }\right)^{1.4} \mu_{\max }\left(l_{\min }\right)}{1-\left(l_{\max } / l_{\min }\right)^{1.4}}
$$

$\mu^{*}$ is an estimation of the solution that would be obtained with an infinitely fine mesh and infinitely small time steps.

Refining the mesh size only without changing the duration of the time steps (mesh convergence) or refining the time steps alone (time convergence) yields the two top curves in Figure G. Using a very fine mesh size with large time steps or very small time steps with a coarse mesh is useless as it does not improve precision but leads to longer computing times.

Figure $\mathbf{\nabla}$ shows that both models give the same evolution of the contact area ratio and of the friction coefficient $\mu$ with time, which validates the transient aspect of the current model. 


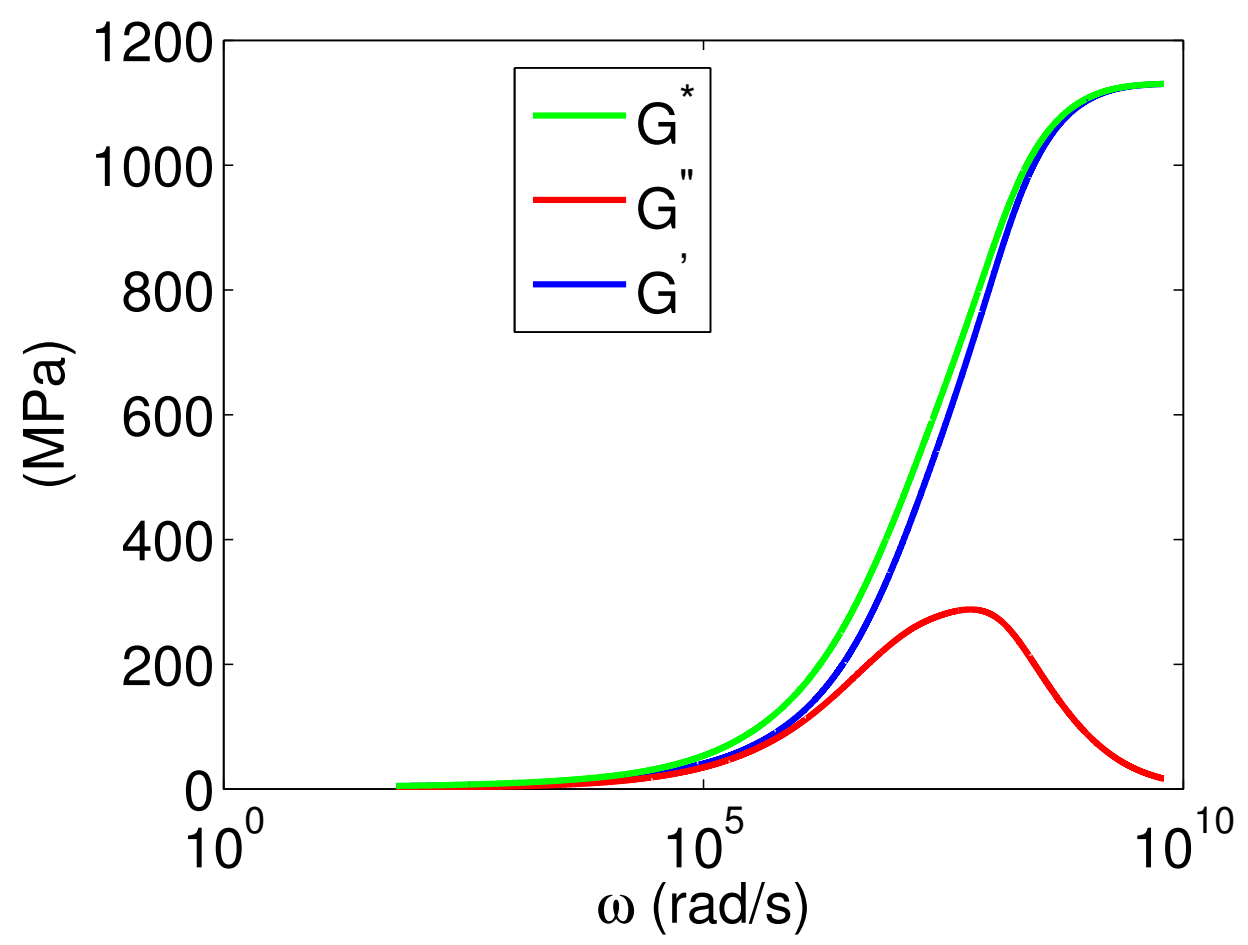

Figure 7: Moduli of the viscoelastic material under periodic excitation. G' is the real part of the modulus, G" the imaginary part and $\mathrm{G}^{*}$ the norm. 

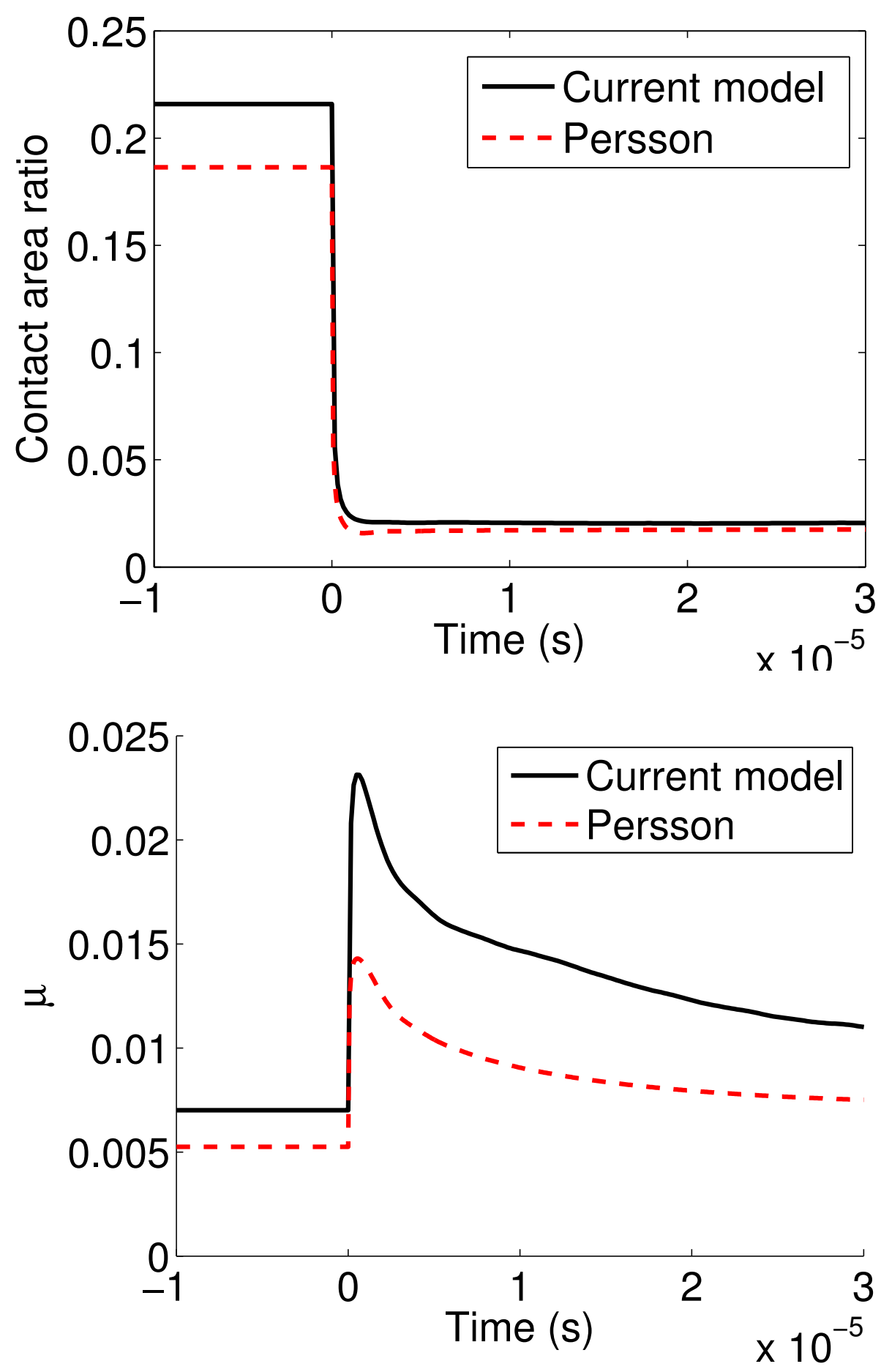

Figure 8: Validation against Persson's model for a velocity step experiment. $V_{0}=0.01 m . s^{-1}, V_{1}=3 m . s^{-1}$ 
Both models give very similar contact area ratios, for a large range of velocities and pressures (see Figures $\mathbf{9}$ and $\mathbf{0}$ ) - Persson's model underestimating the contact area by less than $15 \%$.

Concerning the friction coefficient, results are not as good. The difference between the models is around $30 \%$ for the steady state friction coefficient $\mu_{d y n}$ and up to $50 \%$ for the maximum friction coefficient $\mu_{\max }$ at high velocities. The steady state friction coefficient is approximated by averaging the friction during the last $\approx 3 / 4$ of the total sliding distance, which is $0.5 \mathrm{~mm}$. It is an approximation: as the size of the simulation is $1 \mathrm{~mm}$, sliding for several millimeters would be necessary to reach steady state.

Persson's model gives an exact solution for full contact. That is why at high pressures, when the contact area ratio is close to 1 , the two models agree perfectly. From these results it seems that the reduction factor of 0.45 in Persson's model (which only applies for partial contacts) is slightly too low for the problem considered, although a 30\% difference is still acceptable considering the huge differences between the hypotheses (and the computing time) of the two models.

The velocity at which steady state friction is maximum for the current problem with a nominal pressure of $P_{0}=0.1 \mathrm{MPa}$ is $V_{\max }=0.3 \mathrm{~m} / \mathrm{s}$ for both models. Contrary to the conclusions in [ $\mathrm{z}$ ], we find that a maximum of the friction curve can appear even if $V_{0}$ and $V_{1}$ are both lower than $V_{\max }$. Contrary to the steady state friction $\mu_{d y n}, \mu_{\max }$ is constantly increasing with speed.

Interestingly, both $\mu_{d y n}$ and $\mu_{\max }$ are only slightly dependent on pressure at low pressures. At high pressures there is almost complete contact. In this case the viscoelastic losses do not depend on pressure, leading to decreasing values of the apparent friction coefficient.

\subsection{Tire friction}

Let us now focus on a piece of rubber in a tire. Simulating the tire contact with the current model allows one to estimate the viscoelastic dissipation in a tire and its evolution with pressure and sliding speed. It should be kept in mind that other physical phenomena are likely to occur in tire contact such as interface friction and adhesive forces. Interface friction acts in the tangential direction and therefore does not influence the normal displacements and viscoelastic dissipation due to the normal displacements. Adhesive forces on the other hand may influence it but their effect is out of the scope of the present study.

As the wheel turns, the rubber enters the contact area. At first no sliding occurs: the rubber block is just sheared in the direction of the external force. The direction and intensity of this force depend on the situation: acceleration, breaking, cornering... Rubber then begins to slide. The sliding velocity depends on the force's direction and intensity and on the rotating speed of the wheel.

This sliding history is simulated as follows: first a normal pressure is applied, as rubber enters the contact zone. For $1 \mathrm{~ms}$ no sliding occurs as the rubber block is sheared. Then sliding starts at constant velocity $V \approx 1 \mathrm{~ms}^{-1}$. The material and surface are the same as in the previous paragraph.

As in the previous paragraph, the friction curve goes through a maximum at the the beginning of sliding (see Figure [1). In this case it can be easily explained. During the first millisecond, the contact area increases as the rubber is pushed on the rough surface. When sliding starts, the rubber rises and the contact area decreases, which causes a maximum in the friction coefficient $\mu_{\max }$. As the sliding distance increases, the system reaches a steady state and a constant friction coefficient $\mu_{\text {dyn }}$.

Persson's model gives similar results as the simulation, though it underestimates $\mu_{d y n}$ and especially $\mu_{\max }$ as in the previous paragraph. The evolution with velocity and pressure is the same as in the previous paragraph and thus are not shown here. 

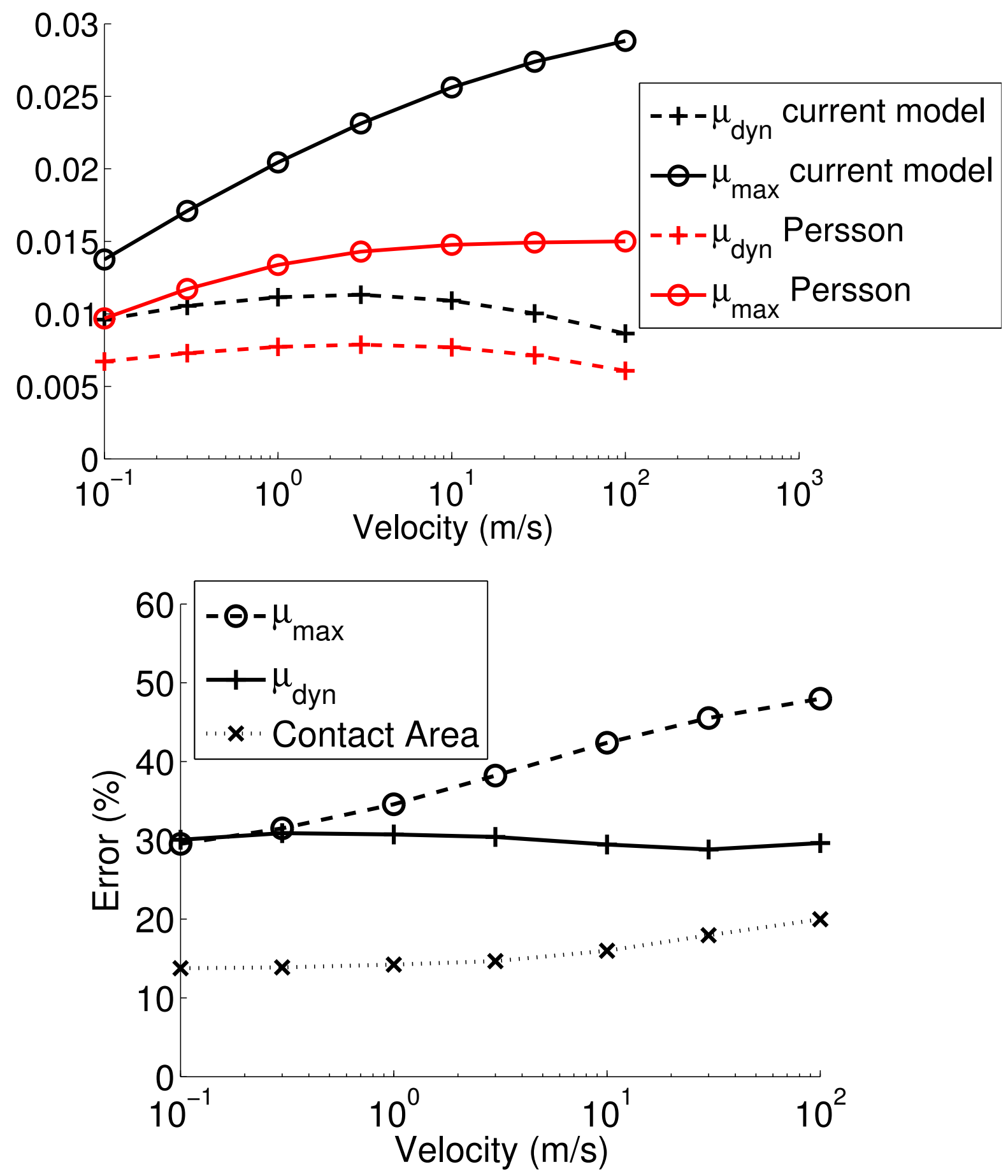

Figure 9: Validation against Persson's model for a velocity step experiment for different velocities. The first figure shows the evolution of $\mu_{d y n}$ and $\mu_{\max }$. The second figure shows the error between the two models, the current model being chosen as reference. 

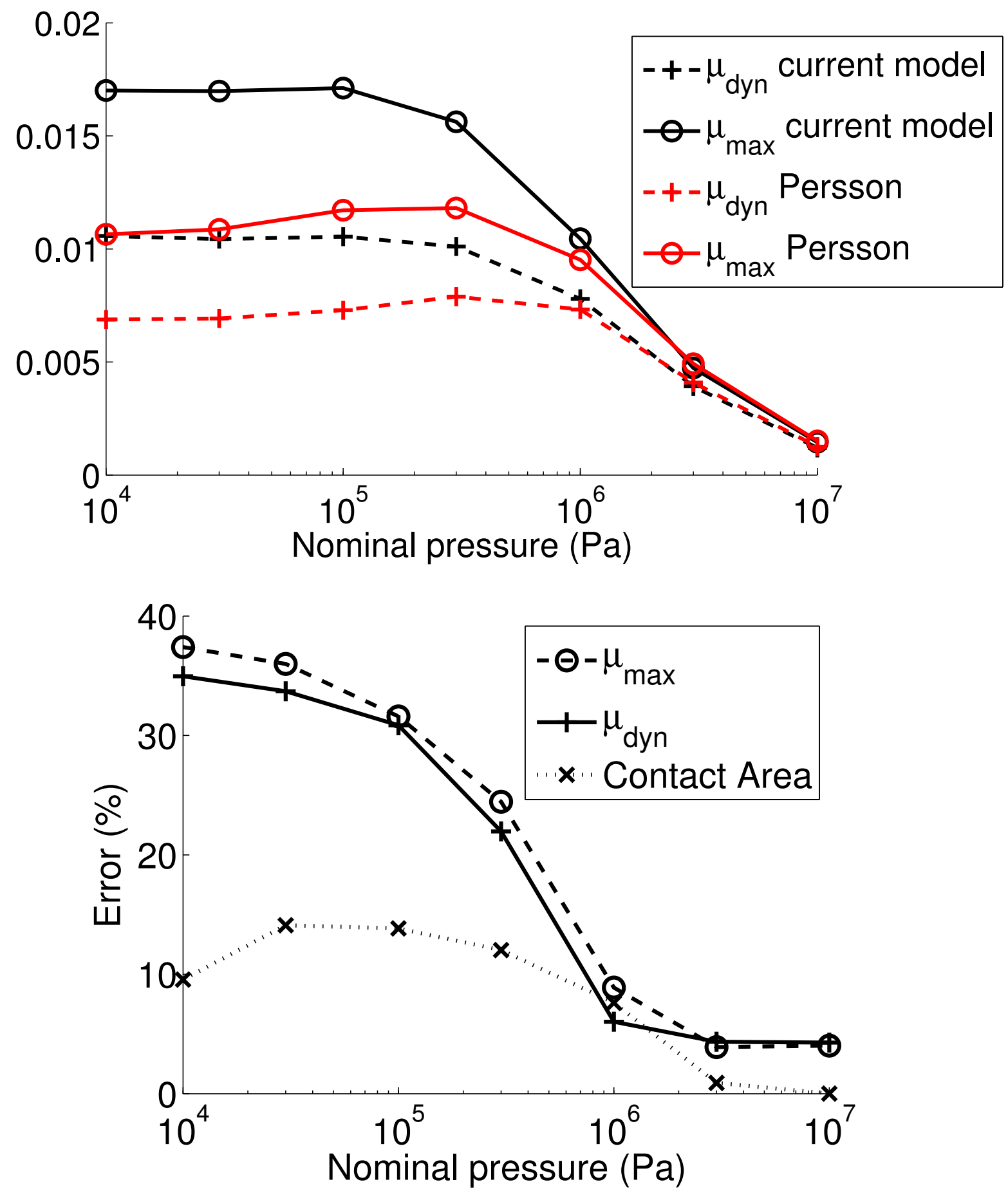

Figure 10: Validation against Persson's model for a velocity step experiment for different nominal pressures. $V_{2}=$ $0.3 \mathrm{~m} / \mathrm{s}$. The first figure shows the evolution of $\mu_{d y n}$ and $\mu_{\max }$. The second figure shows the error between the two models, the current model being chosen as reference. 

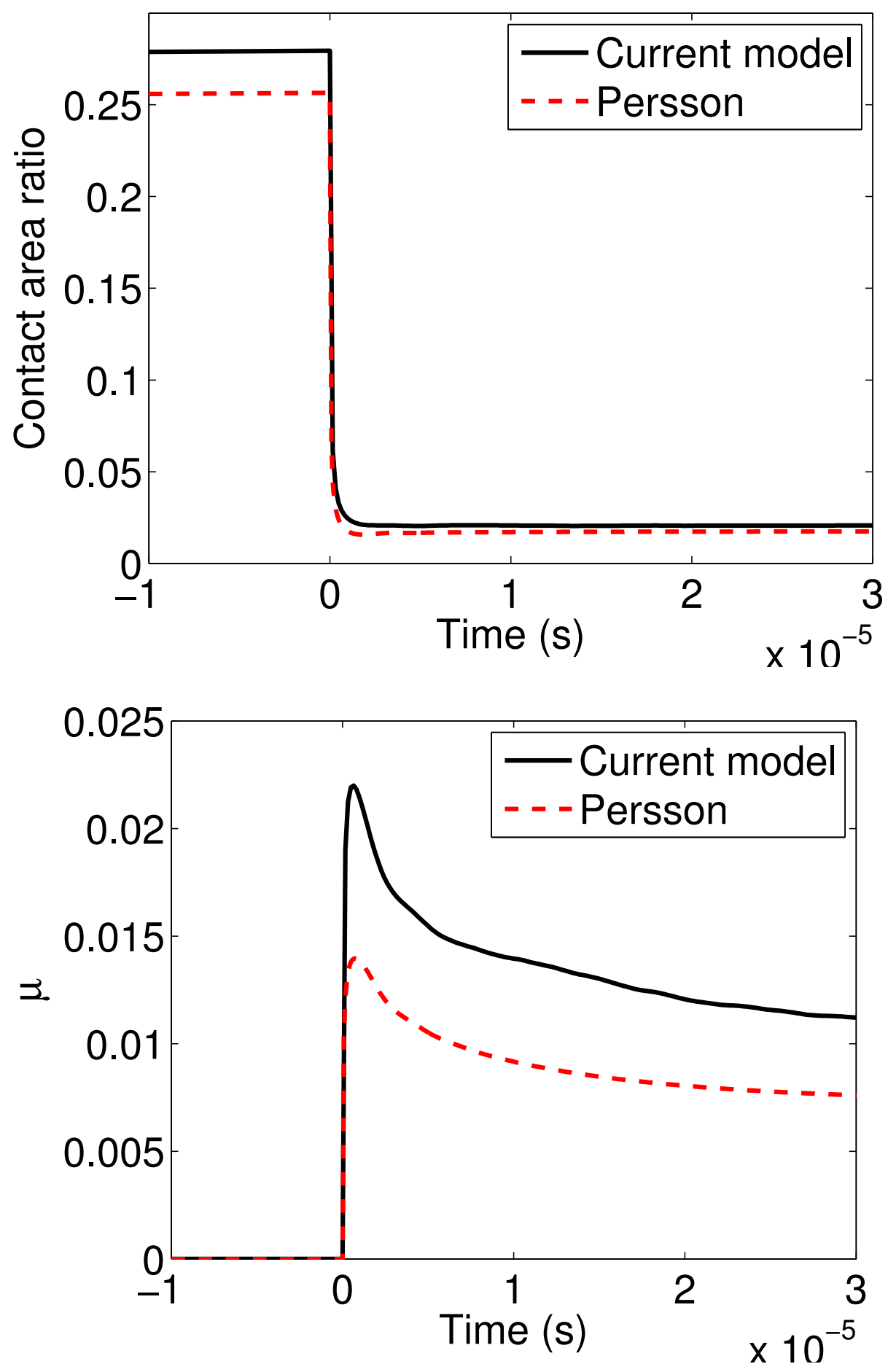

Figure 11: Validation against Persson's model for a tire-like experiment. $P_{0}=0.1 M P a, V_{1}=3 m . s^{-1}$ 


\section{Conclusion}

The model developed in this paper analyses a transient viscoelastic contact using a 'State variable' approach. The model is validated against results from the literature. Comparison with Persson's model shows a good agreement in terms of contact area ratio and friction coefficient. The maximum of friction $\mu_{\max }$ shows a small discrepancy at high velocity, which is not fully understood. Both models are of similar interest for prediction purposes, but lack several features to be representative of real life tire friction on a dry road. The adhesive forces which occur at small scales between the road and the tire lead to an attraction of the two surfaces. This increases the contact area and leads to tangential friction forces at the interface. These two effects will be implemented in the current model. The small strain and small slope hypotheses can also be criticized, but are difficult to overcome without a full Finite Element simulation, which seems extremely costly.

\section{Acknowledgments}

This work was supported by the Manufacture Française de Pneumatiques Michelin. 
[1] J. A. Greenwood and J. B. P. Williamson. Contact of Nominally Flat Surfaces. Proceedings of the Royal Society of London A: Mathematical, Physical and Engineering Sciences, 295(1442):300-319, December 1966.

[2] K. L. Johnson. Contact Mechanics. Cambridge University Press, Cambridge [Cambridgeshire]; New York, 1987.

[3] E. L. Deladi, M. B. de Rooij, and D. J. Schipper. Modelling of static friction in rubber-metal contact. Tribology International, 40(4):588-594, April 2007.

[4] B. N. J. Persson. Theory of rubber friction and contact mechanics. The Journal of Chemical Physics, 115(8):3840-3861, August 2001.

[5] B. N. J. Persson. Contact mechanics for randomly rough surfaces. Surface Science Reports, 61(4):201-227, June 2006.

[6] A. Almqvist, C. Campañá, N. Prodanov, and B. N. J. Persson. Interfacial separation between elastic solids with randomly rough surfaces: Comparison between theory and numerical techniques. Journal of the Mechanics and Physics of Solids, 59(11):2355-2369, November 2011.

[7] M. Scaraggi and B. N. J. Persson. Friction and universal contact area law for randomly rough viscoelastic contacts. Journal of Physics: Condensed Matter, 27(10):105102, March 2015.

[8] B. N. J. Persson and A. I. Volokitin. Theory of rubber friction: Nonstationary sliding. Physical Review B, 65(13):134106, March 2002.

[9] G. Carbone and F. Bottiglione. Asperity contact theories: Do they predict linearity between contact area and load? Journal of the Mechanics and Physics of Solids, 56(8):2555-2572, August 2008.

[10] A. Brandt and A. A. Lubrecht. Multilevel Matrix Multiplication and Fast Solution of Integral Equations. J. Comput. Phys., 90(2):348-370, September 1990.

[11] H. M. Stanley and T. Kato. An FFT-Based Method for Rough Surface Contact. Journal of Tribology, 119(3):481485, July 1997.

[12] Shuangbiao Liu and Qian Wang. Studying Contact Stress Fields Caused by Surface Tractions With a Discrete Convolution and Fast Fourier Transform Algorithm. Journal of Tribology, 124(1):36-45, June 2001.

[13] P. Sainsot and A. A. Lubrecht. Efficient solution of the dry contact of rough surfaces: A comparison of fast Fourier transform and multigrid methods. Proceedings of the Institution of Mechanical Engineers, Part J: Journal of Engineering Tribology, 225(6):441-448, June 2011.

[14] C. Putignano, L. Afferrante, G. Carbone, and G. Demelio. A new efficient numerical method for contact mechanics of rough surfaces. International Journal of Solids and Structures, 49(2):338-343, January 2012.

[15] Giuseppe Carbone and Carmine Putignano. A novel methodology to predict sliding and rolling friction of viscoelastic materials: Theory and experiments. Journal of the Mechanics and Physics of Solids, 61(8):18221834, August 2013.

[16] Carlos Campañá and Martin H. Müser. Practical Green's function approach to the simulation of elastic semiinfinite solids. Physical Review B, 74(7):075420, August 2006.

[17] Nikolay Prodanov, Wolf B. Dapp, and Martin H. Müser. On the Contact Area and Mean Gap of Rough, Elastic Contacts: Dimensional Analysis, Numerical Corrections, and Reference Data. Tribology Letters, 53(2):433-448, December 2013.

[18] Joseph Boussinesq. Application des potentiels à l'étude de l'équilibre et du mouvement des solides élastiques , principalement au calcul des déformations et des pressions que produisent, dans les solides, des efforts quelquonques exercés sur une petite partie de leur surface ou de leur intérieur : mémoire suivi de notes étendues sur divers points de physique mathématique et d'analyse. Gauthier-Villars, 1885.

[19] A. E. H. Love. The Stress Produced in a Semi-Infinite Solid by Pressure on Part of the Boundary. Philosophical Transactions of the Royal Society of London. Series A, Containing Papers of a Mathematical or Physical Character, 228:377-420, 1929.

[20] J. J. Kalker. Three-Dimensional Elastic Bodies in Rolling Contact. Number v. 2 in Solid mechanics and its applications. Kluwer Academic Publishers, Dordrecht ; Boston, 1990.

[21] I. A. Polonsky and L. M. Keer. A numerical method for solving rough contact problems based on the multi-level multi-summation and conjugate gradient techniques. Wear, 231(2):206-219, July 1999.

[22] R. Christensen. Theory of Viscoelasticity: An Introduction. Elsevier, October 1982.

[23] J. C. Simo and T. J. R. Hughes. Computational Inelasticity. Springer Science \& Business Media, May 2006.

[24] Koffi Espoir Koumi, Thibaut Chaise, and Daniel Nelias. Rolling contact of a rigid sphere/sliding of a spherical indenter upon a viscoelastic half-space containing an ellipsoidal inhomogeneity. Journal of the Mechanics and Physics of Solids, 80:1-25, July 2015.

[25] I. F. Kozhevnikov, J. Cesbron, D. Duhamel, H. P. Yin, and F. Anfosso-Lédée. A new algorithm for computing the indentation of a rigid body of arbitrary shape on a viscoelastic half-space. International Journal of Mechanical Sciences, 50(7):1194-1202, July 2008. 
[26] H. P. Yin, J. Cesbron, and Q. H. Bui. A new formulation for solving 3-D time dependent rolling contact problems of a rigid body on a viscoelastic half-space. Mechanics Research Communications, 64:8-14, March 2015.

[27] X. H. Chen and D. W. Wang. Fractal and spectral analysis of aggregate surface profile in polishing process. Wear, 271(11-12):2746-2750, September 2011.

[28] C. G. Panagiotopoulos, V. Mantic, and T. Roubicek. A simple and efficient BEM implementation of quasistatic linear visco-elasticity. International Journal of Solids and Structures, 51(13):2261-2271, June 2014. WOS:000336775400002.

[29] Severino P. C. Marques and Guillermo J. Creus. Computational Viscoelasticity. SpringerBriefs in Applied Sciences and Technology. Springer Berlin Heidelberg, Berlin, Heidelberg, 2012.

[30] Vladislav A. Yastrebov, Guillaume Anciaux, and Jean-François Molinari. From infinitesimal to full contact between rough surfaces: Evolution of the contact area. International Journal of Solids and Structures, 52:83102, January 2015.

[31] Y. Z. Hu and K. Tonder. Simulation of 3-D random rough surface by 2-D digital filter and fourier analysis. International Journal of Machine Tools and Manufacture, 32(1-2):83-90, February 1992. 